\title{
LOCALLY CONFORMALLY KÄHLER STRUCTURES ON UNIMODULAR LIE GROUPS
}

\author{
A. ANDRADA AND M. ORIGLIA
}

\begin{abstract}
We study left-invariant locally conformally Kähler structures on Lie groups, or equivalently, on Lie algebras. We give some properties of these structures in general, and then we consider the special cases when its complex structure is bi-invariant or abelian. In the former case, we show that no such Lie algebra is unimodular, while in the latter, we prove that if the Lie algebra is unimodular, then it is isomorphic to the product of $\mathbb{R}$ and a Heisenberg Lie algebra.
\end{abstract}

\section{INTRODUCTION}

Let $(M, J, g)$ be a $2 n$-dimensional Hermitian manifold and let $\omega$ be its fundamental 2 -form, that is, $\omega(X, Y)=g(J X, Y)$ for any $X, Y$ vector fields on $M$. The manifold $(M, J, g)$ is called locally conformally Kähler (or l.c.K., for short) if $g$ can be rescaled locally, in a neighborhood of any point in $M$, so as to be Kähler, or equivalently, if there exists a closed 1-form $\theta$ such that

$$
d \omega=\theta \wedge \omega .
$$

This 1-form $\theta$ is called the Lee form. This notion was introduced by P. Libermann [17] in 1954, but the geometry of these manifolds was not developed until the 70's, with the work of I. Vaisman. These manifolds are a natural generalization of the class of Kähler manifolds, and they have been much studied by many authors (see for instance [8, 22, 25]). According to [9], a locally conformally Kähler manifold is in the class $\mathcal{W}_{4}$ of the Gray-Hervella classification of almost Hermitian manifolds. An important class of l.c.K. metrics is given by those whose Lee form is parallel with respect to the Levi-Civita connection. These l.c.K. structures are called Vaisman, and their existence imposes topological and cohomological restrictions on the underlying Hermitian manifold (see for instance $[25])$.

We will consider locally conformally Kähler structures on solvmanifolds, that is, compact quotients $\Gamma \backslash G$ where $G$ is a simply connected solvable Lie group and $\Gamma$ is a lattice in $G$, which are induced by left-invariant locally conformally Kähler structures on the Lie group. These structures have been the subject of study in several recent papers. For instance, it was shown in [21] that if a non-toral nilmanifold admits an invariant locally conformally Kähler structure, then it is a quotient of $\mathbb{R} \times H_{2 n+1}$, where $H_{2 n+1}$ is the $(2 n+1)$-dimensional Heisenberg Lie group. In [22] it was proved that any invariant locally conformally Kähler structure on a solvmanifold such that $\omega=-\theta \wedge J \theta+d(J \theta)$ is in fact Vaisman. According to [19] this condition is related to the existence of a potential for the l.c.K. metric. In [13] it is proved the non-existence of Vaisman metrics on solvmanifolds satisfying certain cohomological conditions.

In this article we study l.c.K. structures on Lie algebras with two special kinds of complex structures. First, we take into account bi-invariant complex structures on Lie algebras, i.e., an endomorphism $J$ of a Lie algebra $\mathfrak{g}$ that satisfies

$$
J^{2}=-I, \quad J[X, Y]=[X, J Y] \text { for all } X, Y \in \mathfrak{g} .
$$

2010 Mathematics Subject Classification. 53C15, 53B35, 53C30.

Key words and phrases. Hermitian metric, locally conformally Kähler metric, abelian complex structure.

The authors were partially supported by CONICET, ANPCyT and SECyT-UNC (Argentina). 
This condition holds if and only if both left- and right-translations on the corresponding simply connected (real) Lie group are holomorhic, or equivalently, this Lie group is in fact a complex Lie group.

The other special kind of complex structures that we consider is given by the so called abelian complex structures. Recall that an abelian complex structure on a Lie algebra $\mathfrak{g}$ is an endomorphism $J$ of $\mathfrak{g}$ that satisfies

$$
J^{2}=-I, \quad[J X, J Y]=[X, Y] \text { for all } X, Y \in \mathfrak{g},
$$

or equivalently, the $i$-eigenspace of $J$ in $\mathfrak{g}^{\mathbb{C}}$ is an abelian subalgebra of $\mathfrak{g}^{\mathbb{C}}$. There are well known obstructions for the existence of abelian complex structures. For instance, if the Lie algebra $\mathfrak{g}$ admits such a structure, then $\mathfrak{g}$ has abelian commutator (i.e., $\mathfrak{g}$ is two-step solvable), and the center of $\mathfrak{g}$ is $J$-invariant, among other properties (see Lemma 2.5). These structures are very important in several areas of geometry and they have been studied by many authors recently (see for instance $[5,7,14])$.

The outline of this article is as follows. In Section 2 we review some known results about l.c.K. manifolds and left-invariant complex structures on Lie groups. In Section 3 we determine some properties of Lie algebras endowed with an l.c.K. or Vaisman structure. Next, in Section 4 we prove that there exists no unimodular Lie algebra $\mathfrak{g}$ equipped with an l.c.K. structure $(J,\langle\cdot, \cdot\rangle)$ where $J$ is bi-invariant (Theorem 4.1). Finally, in Section 5 we prove that if $(\mathfrak{g}, J,\langle\cdot, \cdot\rangle)$ is l.c.K. with an abelian complex structure $J$ and $\mathfrak{g}$ is unimodular then $\mathfrak{g} \simeq \mathbb{R} \times \mathfrak{h}_{2 n+1}$, where $\mathfrak{h}_{2 n+1}$ is the $(2 n+1)$-dimensional Heisenberg Lie algebra. Moreover, there is only one, up to equivalence, monoparametric family $\left(J_{0},\langle\cdot, \cdot\rangle_{\lambda}\right), \lambda>0$, of l.c.K. structures on this Lie algebra, where the metrics $\langle\cdot, \cdot\rangle_{\lambda}$ are pairwise non-isometric.

\section{Preliminaries}

\subsection{Locally conformally Kähler manifolds.}

A Hermitian metric on an almost complex manifold $(M, J)$ is a Riemannian metric $g$ such that $g(X, Y)=g(J X, J Y)$ for any vector fields $X, Y$ on $M$. In this case $(M, J, g)$ is called an almost Hermitian manifold. When the almost complex structure $J$ is integrable (i.e., $(M, J)$ is a complex manifold), then $(M, J, g)$ is called a Hermitian manifold.

Given an almost Hermitian manifold $(M, J, g)$, the fundamental 2-form is defined by $\omega(X, Y)=$ $g(J X, Y)$ for any vector fields $X, Y$ on $M$.

A Kähler metric on a complex manifold $(M, J)$ is a Hermitian metric $g$ whose fundamental 2 -form $\omega$ is closed, that is, $d \omega=0$. Then $M$ is called a Kähler manifold.

Kähler manifolds are by far the most important Hermitian manifolds. Nevertheless, this condition might be very restrictive in some cases, and therefore, weaker conditions are studied. One way to do so is to consider Hermitian manifolds whose metric is locally conformal to a Kähler metric.

The Hermitian manifold $(M, J, g)$ is locally conformally Kähler (l.c.K.) if there exists an open covering $\left\{U_{i}\right\}_{i \in I}$ of $M$ and a family $\left\{f_{i}\right\}_{i \in I}$ of $C^{\infty}$-functions, $f_{i}: U_{i} \rightarrow \mathbb{R}$, such that each local metric

$$
g_{i}=\left.\exp \left(-f_{i}\right) g\right|_{U_{i}}
$$

is Kähler. Also $(M, J, g)$ is globally conformally Kähler (g.c.K.) if there exists a $C^{\infty}$-function, $f: M \rightarrow \mathbb{R}$, such that the metric $\exp (f) g$ is Kähler.

We recall an important result which characterizes l.c.K. manifolds in terms of its fundamental form (see [8] for a proof). 
Theorem $2.1([16])$. The Hermitian manifold $(M, J, g)$ is l.c.K. if and only if there exists a closed 1-form $\theta$ globally defined on $M$ such that

$$
d \omega=\theta \wedge \omega .
$$

Moreover, $(M, J, g)$ is globally conformally Kähler if and only the 1-form $\theta$ in (2) is exact.

Remarks. (i) A simply connected l.c.K. manifold is g.c.K., in particular the universal cover of a l.c.K. manifold is g.c.K.

(ii) An l.c.K. manifold $(M, J, g)$ is Kähler if and only if $\theta=0$. Indeed, $\theta \wedge \omega=0$ and $\omega$ nondegenerate imply $\theta=0$.

(iii) It is known that if $(M, J, g)$ is a Hermitian manifold with $\operatorname{dim} M \geq 6$ such that (2) holds for some 1 -form $\theta$, then $\theta$ is automatically closed, therefore $M$ is l.c.K.

The 1-form $\theta$ of the previous theorem is called the Lee form and it was introduced by H. C. Lee in [16]. The Lee form is uniquely determined by the following formula:

$$
\theta=-\frac{1}{n-1}(\delta \omega) \circ J
$$

where $\omega$ is the fundamental 2 -form, $\delta$ is the codifferential and $2 n$ is the dimension of $M$. In general this formula is used to define the Lee form of any almost Hermitian manifold.

Example 2.2. The Hopf manifolds are examples of locally conformally Kähler manifolds which are not g.c.K. Let $\lambda \in \mathbb{C},|\lambda| \neq 1$ and $\triangle_{\lambda}$ be the cyclic group generated by transformations $z \mapsto \lambda z$ of $\mathbb{C}^{n}-\{0\}$. The quotient space $C H_{\lambda}^{n}=\left(\mathbb{C}^{n}-\{0\}\right) / \triangle_{\lambda}$ is a complex manifold and it is called Hopf's complex manifold. It can be seen that $C H_{\lambda}^{n}$ is diffeomorphic to $S^{1} \times S^{2 n-1}$. Particularly $C H_{\lambda}^{n}$ is compact and its first Betti number is $b_{1}\left(C H_{\lambda}^{n}\right)=1$. Since all odd Betti numbers of a compact Kähler manifold are even, it follows that $C H_{\lambda}^{n}$ cannot admit a Kähler metric.

We consider now the Hermitian metric on $\mathbb{C}^{n}-\{0\}$

$$
h=\sum \frac{d z_{j} \otimes d \bar{z}_{j}}{|z|^{2}}
$$

and canonical complex structure $J$. This metric is invariant by $\triangle_{\lambda}$, then it induces a Hermitian metric on $C H_{\lambda}^{n}$ which is called the Boothby's metric. This Hermitian structure on $C H_{\lambda}^{n}$ is in fact l.c.K. and, moreover, $\theta$ is parallel with respect to Levi Civita connection. The l.c.K. manifolds with this property are a special case of l.c.K. manifolds.

Let $(M, J, g)$ be an l.c.K. manifold. The metric $g$ on $M$ is called Vaisman if the Lee form $\theta$ is parallel with respect to the Levi-Civita connection of $(M, g)$. A Vaisman manifold is an l.c.K. manifold with a Vaisman metric. It is known that Vaisman manifolds have some special properties which do not necessarily hold in l.c.K. manifolds. For example, the first Betti number of a Vaisman manifold is odd $([26,12])$, while the Oeljeklaus-Toma manifolds are examples of l.c.K. manifolds with even first Betti number ([18]).

\subsection{Complex structures on Lie algebras.}

A left-invariant almost complex structure $J$ on a Lie group $G$ is a $(1,1)$-differential tensor such that $J_{g}: T_{g} G \rightarrow T_{g} G$ is an endomorphism, $J_{g}^{2}=-$ Id for all $g \in G$ and left-traslations are holomorphic. As usual, the almost complex structure $J$ on $G$ is called integrable if

$$
[J X, J Y]-[X, Y]-J([J X, Y]+[X, J Y])=0,
$$

for any $X, Y$ vector fields on $G$. In this case, $J$ is called a left-invariant complex structure and $(G, J)$ is a complex manifold. 
A left-invariant (almost) complex structure is determined by its value on the identity of $G$, and therefore it is possible to define an (almost) complex structure on the Lie algebra $\mathfrak{g}$ of $G$. Namely, a complex structure $J$ on a Lie algebra $\mathfrak{g}$ is an endomorphism $J: \mathfrak{g} \rightarrow \mathfrak{g}$ satisfying $J^{2}=-\mathrm{Id}$ and

$$
[J X, J Y]-[X, Y]-J([J X, Y]+[X, J Y])=0,
$$

for any $X, Y \in \mathfrak{g}$.

In this article we will be interested in two special kinds of almost complex structures on Lie algebras, namely bi-invariant complex structure and abelian complex structures. An almost complex structure $J$ on $\mathfrak{g}$ is called bi-invariant if

$$
J[X, Y]=[X, J Y], \text { for all } X, Y \in \mathfrak{g},
$$

and it is called abelian if

$$
[J X, J Y]=[X, Y], \text { for all } X, Y \in \mathfrak{g} .
$$

Remark 2.3. Note that in both cases the almost complex structure is automatically integrable. Also, a complex structure on $\mathfrak{g}$ cannot be bi-invariant and abelian at the same time, unless $\mathfrak{g}$ is an abelian Lie algebra.

Remark 2.4. In general, right-traslations are not holomorphic on a Lie group $G$ with a leftinvariant complex structure $J$. This holds only when $G$ is a complex Lie group with the holomorphic structure given by $J$, or equivalently, $J$ is bi-invariant.

Next, we include some properties about abelian complex structures in the following lemma (see $[1,4,20]$ for their proof).

Lemma 2.5. Let $\mathfrak{g}$ be a Lie algebra with an abelian complex structure $J$ and $\mathfrak{z}(\mathfrak{g})$ its center. Then

(1) $J \mathfrak{z}(\mathfrak{g})=\mathfrak{z}(\mathfrak{g})$.

(2) $\mathfrak{g}^{\prime} \cap J \mathfrak{g}^{\prime} \subset \mathfrak{z}\left(\mathfrak{g}^{\prime}+J \mathfrak{g}^{\prime}\right)$.

(3) The codimension of $[\mathfrak{g}, \mathfrak{g}]$ is at least 2 , unless $\mathfrak{g}$ is isomorphic to $\mathfrak{a} \mathfrak{f} \mathfrak{f}(\mathbb{R})$ (the only 2-dimensional non-abelian Lie algebra).

(4) $\mathfrak{g}^{\prime}$ is abelian, therefore $\mathfrak{g}$ is 2-step solvable.

A rich family of Lie algebras with (abelian) complex structures is obtained by considering a finite dimensional real associative algebra $\mathcal{A}$ and $\mathfrak{a f f}(\mathcal{A})$ the vector space $\mathcal{A} \oplus \mathcal{A}$ equipped with the Lie bracket given by

$$
\left[(a, b),\left(a^{\prime}, b^{\prime}\right)\right]=\left(a a^{\prime}-a^{\prime} a, a b^{\prime}-a^{\prime} b\right), \quad a, b, a^{\prime}, b^{\prime} \in \mathcal{A} .
$$

If $J$ is the endomorphism of $\mathfrak{a f f}(\mathcal{A})$ defined by

$$
J(a, b)=(b,-a), \quad a, b \in \mathcal{A},
$$

then it is easy to see that $J$ is a complex structure on $\mathfrak{a f f}(\mathcal{A})$. This complex structure is called standard. Moreover, when $\mathcal{A}$ is commutative, $J$ is abelian. We prove next a result about $\mathfrak{a f f}(\mathcal{A})$ that will be used later.

Lemma 2.6. If $\mathcal{A}$ is an associative commutative algebra and $\mathfrak{a f f}(\mathcal{A})$ is unimodular, then $\mathcal{A}$ is nilpotent. Therefore $\mathfrak{a f f}(\mathcal{A})$ is a nilpotent Lie algebra.

Proof. Suppose that $\mathcal{A}$ is not nilpotent, then there exists $0 \neq e \in \mathcal{A}$ such that $e^{2}=e$. We consider $(e, 0) \in \mathfrak{a f f}(\mathcal{A})$ and we compute $\operatorname{ad}_{(e, 0)}(x, y)=(0, e y)=\left(0, l_{e}(y)\right)$ where $l_{e}$ is the left-multiplication by $e$. Therefore the matrix of $\operatorname{ad}_{(e, 0)}$ is of the form

$$
\operatorname{ad}_{(e, 0)}=\left(\begin{array}{ll}
0 & 0 \\
0 & l_{e}
\end{array}\right)
$$


Since $l_{e}^{2}=l_{e}$ and $l_{e} \neq 0$, there exists a basis of $\mathcal{A}$ such that

$$
l_{e}=\left(\begin{array}{ll}
I & 0 \\
0 & 0
\end{array}\right)
$$

and therefore $\operatorname{tr}\left(\operatorname{ad}_{(e, 0)}\right) \neq 0$.

Remark 2.7. With a similar proof, one can show that if $\mathcal{A}$ is an associative algebra with identity, then $\mathfrak{a f f}(\mathcal{A})$ is not unimodular.

\section{LEFT INVARIANT L.C.K. METRICS ON LIE GROUPS}

Let $G$ be a Lie group with a left-invariant complex structure $J$ and a left-invariant metric $g$. If $(G, J, g)$ satisfies the l.c.K. condition $(2)$, then $(J, g)$ is called a left-invariant l.c.K. structure on the Lie group $G$. That is, there exists a closed 1-form $\theta$ on $G$ such that $d \omega=\theta \wedge \omega$. We will see next that the Lee form $\theta$ is left-invariant. Therefore the Lee form $\theta$ is determined by its value in the identity.

Proposition 3.1. Let $G$ be a Lie group with a left-invariant l.c.K. structure $(J, g)$, with $\theta$ the associated Lee form. Then $\theta$ is left-invariant.

Proof. Recall that if $\alpha$ is any left-invariant form on $G$, then $d \alpha, * \alpha$ and $\delta \alpha= \pm * \circ d \circ * \alpha$ are also left-invariant. Since $J$ is left-invariant, the claim follows from (3).

This fact allows us to define l.c.K. structures on Lie algebras.

Let $\mathfrak{g}$ a Lie algebra, $J$ a complex structure and $\langle\cdot, \cdot\rangle$ a Hermitian inner product on $\mathfrak{g}$, with $\omega$ its fundamental 2 -form. The triple $(\mathfrak{g}, J,\langle\cdot, \cdot\rangle)$ is called locally conformally Kähler (l.c.K.) if there exists $\theta \in \mathfrak{g}^{*}$, with $d \theta=0$, such that ${ }^{1}$

$$
d \omega=\theta \wedge \omega .
$$

A Lie algebra $\mathfrak{g}$ with a Hermitian structure $(J,\langle\cdot, \cdot\rangle)$ is Vaisman if $(\mathfrak{g}, J,\langle\cdot, \cdot\rangle)$ is l.c.K. and the Lee form is parallel (see Proposition 3.5 below).

Example 3.2. Let $\mathfrak{g}=\mathbb{R} \times \mathfrak{h}_{2 n+1}$, where $\mathfrak{h}_{2 n+1}$ is the $(2 n+1)$-dimensional Heisenberg Lie algebra. There is a basis $\left\{X_{1}, \ldots, X_{n}, Y_{1}, \ldots, Y_{n}, Z_{1}, Z_{2}\right\}$ of $\mathfrak{g}$ with Lie brackets given by $\left[X_{i}, Y_{i}\right]=Z_{1}$ for $i=1, \ldots, n$ and $Z_{2}$ in the center. We define a metric $\langle\cdot, \cdot\rangle$ on $\mathfrak{g}$ such that the basis above is orthonormal. Let $J_{0}$ be an almost complex structure given by:

$$
J_{0} X_{i}=Y_{i}, \quad J_{0} Z_{1}=-Z_{2} \text { for } i=1, \ldots, n .
$$

It is easily seen that $J_{0}$ is a complex structure on $\mathfrak{g}$. Let $\left\{x^{i}, y^{i}, z^{1}, z^{2}\right\}$ be the 1 -forms dual to $\left\{X_{i}, Y_{i}, Z_{1}, Z_{2}\right\}$ respectively. Then the fundamental form is:

$$
\omega=\sum_{i=1}^{n}\left(x^{i} \wedge y^{i}\right)-z^{1} \wedge z^{2} .
$$

Thus $d \omega$ is:

$$
d \omega=z^{2} \wedge \omega
$$

and therefore $\left(\mathfrak{g}, J_{0},\langle\cdot, \cdot\rangle\right)$ is l.c.K. It can be seen that the Lee form $\theta=z^{2}$ is parallel, hence the metric is Vaisman. This example appeared in [6].

\footnotetext{
${ }^{1}$ Recall that if $\theta \in \mathfrak{g}^{*}$ and $\omega \in \Lambda^{2} \mathfrak{g}^{*}$, then their exterior derivatives $d \theta \in \Lambda^{2} \mathfrak{g}^{*}$ and $d \omega \in \Lambda^{3} \mathfrak{g}^{*}$ are given by $d \theta(X, Y)=-\theta([X, Y]), \quad d \omega(X, Y, Z)=-\omega([X, Y], Z)-\omega([Y, Z], X)-\omega([Z, X], Y)$,
}

for any $X, Y, Z \in \mathfrak{g}$. 
It is known that $\mathfrak{g}$ is the Lie algebra of the Lie group $\mathbb{R} \times H_{2 n+1}$, where $H_{2 n+1}$ is the group of all matrices with real coefficients which have the following form:

$$
P=\left(\begin{array}{ccc}
1 & A & c \\
0 & I_{n} & B^{t} \\
0 & 0 & 1
\end{array}\right) \quad c \in \mathbb{R}, I_{n}=i d_{n \times n} .
$$

where $A=\left(a_{1}, \ldots, a_{n}\right) \in \mathbb{R}^{n}, B=\left(b_{1}, \ldots, b_{n}\right) \in \mathbb{R}^{n}$ and $c \in \mathbb{R}$. Let $\Gamma \subset H_{2 n+1}$ be the subgroup of all matrices with integer coefficients. Then $\Gamma \backslash H_{2 n+1}$ is compact and the nilmanifold $N=$ $S^{1} \times \Gamma \backslash H_{2 n+1}$ admits a l.c.K. structure which is Vaisman.

Remark. The complex structure $J_{0}$ defined in the previous example is abelian. Moreover, it was proved in [4] that if $J$ is a complex structure on a Lie algebra $\mathfrak{g}$ with $\operatorname{dim} \mathfrak{g}^{\prime}=1$, then $J$ is abelian.

Example 3.3. In [3] the following example of a l.c.K. solvmanifold was given. Let $\mathfrak{g}$ be the 4-dimensional solvable Lie algebra given by

$$
\begin{gathered}
\mathfrak{g}=\operatorname{span}\{A, X, Y, Z\} \\
{[A, X]=X, \quad[A, Y]=-Y, \quad[X, Y]=Z .}
\end{gathered}
$$

Let $\{\alpha, x, y, z\}$ be the dual basis of $\{A, X, Y, Z\}$. We can check by direct computation that

$$
d \alpha=0, \quad d x=-\alpha \wedge x, \quad d y=\alpha \wedge y, \quad d z=-x \wedge y .
$$

Let $\langle\cdot, \cdot\rangle$ be a inner product on $\mathfrak{g}$ such that $\{A, X, Y, Z\}$ is an orthonormal basis. If we define $J$ by

$$
J A=Y, \quad J Z=X,
$$

then $(\mathfrak{g}, J,\langle\cdot, \cdot\rangle)$ is Hermitian with the fundamental 2 -form $\omega$ given by

$$
\omega=\alpha \wedge y+z \wedge x
$$

Therefore we obtain

$$
d \omega=-\alpha \wedge \omega .
$$

Hence it is l.c.K. with Lee form $\theta=-\alpha$. This metric is not Vaisman, since $\alpha$ is not parallel (see also Lemma 3.5 below). It was proved in [3] that the associated simply connected solvable Lie group $G$ admits a lattice $\Gamma$ and therefore the solvmanifold $\Gamma \backslash G$ admits a l.c.K. structure. It is proved in [11] that this l.c.K. solvmanifold is holomorphically homothetic to the Inoue surface $S o l_{1}^{4} / \Gamma$ equipped with the locally conformal Kähler structure constructed by Tricerri in [23].

Now we study some properties about Lie algebras equipped with a l.c.K or Vaisman structure.

Let $(\mathfrak{g}, J,\langle\cdot, \cdot\rangle)$ be l.c.K. and suppose that $\mathfrak{g}$ is not Kähler, that is, $d \omega=\theta \wedge \omega$ where $\theta$ is closed and $\theta \neq 0$. Then the codimension of $\operatorname{ker} \theta$ is 1 and then we can choose

$$
A \in(\operatorname{ker} \theta)^{\perp} \text { such that } \theta(A)=1,
$$

and therefore $\mathfrak{g}$ can be decomposed orthogonally as

$$
\mathfrak{g}=\operatorname{span}\{A\} \oplus \operatorname{ker} \theta, \quad \text { with } \quad \mathfrak{g}^{\prime} \subset \operatorname{ker} \theta .
$$

Note that since $\theta \neq 0, \mathfrak{g}$ cannot be a semisimple Lie algebra. Since $J$ is skew-symmetric we obtain $\langle J A, A\rangle=0$ and therefore $J A \in \operatorname{ker} \theta$. If $W$ is the orthogonal complement of $\operatorname{span}\{J A\}$ in $\operatorname{ker} \theta$, we have

$$
\mathfrak{g}=\operatorname{span}\{A, J A\} \oplus^{\perp} W
$$

and $W$ is invariant by $J$. 
Note that the Lee form can be written as

$$
\theta(X)=\frac{\langle X, A\rangle}{|A|^{2}} \text { for all } X \in \mathfrak{g} .
$$

Lemma 3.4. If $(\mathfrak{g}, J,\langle\cdot, \cdot\rangle)$ is l.c.K. then $J \circ \operatorname{ad}_{J A}$ is symmetric.

Proof. For any $X, Y \in \mathfrak{g}$ we compute

$$
\begin{aligned}
d \omega(J A, X, Y) & =-\omega([J A, X], Y)-\omega([X, Y], J A)-\omega([Y, J A], X) \\
& =-\langle J[J A, X], Y\rangle-\langle J[X, Y], J A\rangle-\langle J[Y, J A], X\rangle \\
& =-\langle J[J A, X], Y\rangle-\langle J[Y, J A], X\rangle .
\end{aligned}
$$

On the other hand, using (10), we obtain

$$
\begin{aligned}
\theta \wedge \omega(J A, X, Y) & =\theta(X) \omega(Y, J A)+\theta(Y) \omega(J A, X) \\
& =\frac{\langle A, X\rangle}{|A|^{2}}\langle Y, A\rangle-\frac{\langle A, Y\rangle}{|A|^{2}}\langle A, X\rangle \\
& =0 .
\end{aligned}
$$

It follows from (6) that $\langle J[J A, X], Y\rangle=\langle J[J A, Y], X\rangle$ for all $X, Y \in \mathfrak{g}$, hence $J \circ$ ad $_{J A}$ is symmetric.

Now we consider a Vaisman structure $(J,\langle\cdot, \cdot\rangle)$ on $\mathfrak{g}$, with $\theta$ its parallel Lee form. In this context the Vaisman condition can be characterized as follows.

Lemma 3.5. Let $(\mathfrak{g}, J,\langle\cdot, \cdot\rangle)$ be l.c.K. and let $A \in \mathfrak{g}$ be as in $(7)$. Then $(\mathfrak{g}, J,\langle\cdot, \cdot\rangle)$ is Vaisman if and only if $\operatorname{ad}_{A}$ is skew-symmetric.

Proof. Recall first that the Levi-Civita connection $\nabla$ of a left-invariant Riemannian metric on a Lie group is itself left-invariant, that is, $\nabla_{X} Y$ is a left-invariant vector field whenever $X, Y$ are left-invariant. Similarly, $\nabla T$ is a left-invariant tensor if $T$ is a left-invariant tensor. In particular, if $\eta$ is a left-invariant 1-form, we have that $\left(\nabla_{X} \eta\right)(Y)=-\eta\left(\nabla_{X} Y\right)$ for $X, Y$ left-invariant vector fields.

Let us now compute $\nabla \theta$, where $\nabla$ is the Levi-Civita connection on $\mathfrak{g}$ associated to $\langle\cdot, \cdot\rangle$ and $\theta$ is the Lee form. Given $X, Y \in \mathfrak{g}$ we have that

$$
\left(\nabla_{X} \theta\right)(Y)=-\theta\left(\nabla_{X} Y\right)=-\frac{\left\langle\nabla_{X} Y, A\right\rangle}{|A|^{2}}=-\frac{1}{2|A|^{2}}\{\langle[X, Y], A\rangle-\langle[Y, A], X\rangle+\langle[A, X], Y\rangle\}
$$

Since $A$ is orthogonal to $\mathfrak{g}^{\prime}$ we obtain:

$$
\left(\nabla_{X} \theta\right)(Y)=-\frac{1}{2|A|^{2}}\{\langle[A, Y], X\rangle+\langle[A, X], Y\rangle\}
$$

Therefore $\left(\nabla_{X} \theta\right)(Y)=0$ if and only if $\langle[A, Y], X\rangle=-\langle[A, X], Y\rangle$ for all $X, Y \in \mathfrak{g}$.

Remark 3.6. Let $H$ be a Lie group equipped with a left-invariant metric $h$. Recall that the endomorphism $\operatorname{ad}_{A}$ of its Lie algebra $\mathfrak{h}$ is skew-symmetric with respect to $h_{e}$ if and only if the left-invariant vector field on $H$ determined by $A$ is Killing. 


\section{L.C.K. STRUCTURES With Bi-INVARiant COMPleX STRUCTURE}

In this section we consider a Lie algebra equipped with an l.c.K. structure such that its complex structure is bi-invariant. Equivalently, we are considering left-invariant l.c.K. metrics on complex Lie groups. The aim of this section is to prove the following result, where we show that in each even (real) dimension, there is only one Lie algebra admitting such metrics.

Theorem 4.1. Let $(J,\langle\cdot, \cdot\rangle)$ be an l.c.K. structure on $\mathfrak{g}$ with a bi-invariant complex structure $J$. Then $\mathfrak{g} \simeq \mathbb{R}^{2} \ltimes \mathbb{R}^{2 n}$, a J-invariant and orthogonal sum, where $\mathbb{R}^{2}$ is generated by $A, B$ with $[A, B]=0, J A=B,[A, X]=-\frac{1}{2} X$ and $[B, X]=-\frac{1}{2} J X$ for all $X \in \mathbb{R}^{2 n}$.

In order to prove this theorem, we recall the following well known result concerning the existence of Kähler metrics on complex Lie groups.

Lemma 4.2. If $(\mathfrak{g}, J,\langle\cdot, \cdot\rangle)$ is Kähler with $J$ bi-invariant, then $\mathfrak{g}$ is abelian.

Proof of Theorem. The fact that $J$ is bi-invariant implies that

$$
J \mathfrak{g}^{\prime}=\mathfrak{g}^{\prime} .
$$

Recall from (8) the orthogonal decomposition

$$
\mathfrak{g}=\operatorname{span}\{A\} \oplus \operatorname{ker} \theta .
$$

Lemma 4.3. The endomorphism $\operatorname{ad}_{A}: \mathfrak{g} \rightarrow \mathfrak{g}$ is symmetric.

Proof. Since $J$ is bi-invariant we have that $J \circ \operatorname{ad}_{J A}=-\operatorname{ad}_{A}$, and it follows from Lemma 3.4 that $\operatorname{ad}_{A}$ is symmetric.

Next, from (9) we have

$$
\mathfrak{g}=\operatorname{span}\{A, J A\} \oplus^{\perp} W
$$

where $\mathfrak{g}^{\prime} \subset \operatorname{ker} \theta=\operatorname{span}\{J A\} \oplus W$ and $W$ is $J$-invariant. It is easy to see that $\mathfrak{g}^{\prime} \subset W$. Actually $\mathfrak{g}^{\prime}=W$, since for $X \in W$ we have $d \omega(A, X, J X)=-2\langle[A, X], X\rangle$ and $\theta \wedge \omega(A, X, J X)=|X|^{2}$, therefore

$$
-2\langle[A, X], X\rangle=|X|^{2}
$$

this implies that $\mathfrak{g}^{\prime}=W$. Then we obtain

$$
\mathfrak{g}=\operatorname{span}\{A, J A\} \oplus^{\perp} \mathfrak{g}^{\prime},
$$

with $\mathfrak{g}^{\prime} J$-invariant.

On the other hand $\left(\mathfrak{g}^{\prime},\left.J\right|_{\mathfrak{g}^{\prime}},\langle\cdot, \cdot\rangle\right)$ is Kähler, since the fundamental form of $\mathfrak{g}^{\prime}$ is the restriction of $\omega$ to $\mathfrak{g}^{\prime} \times \mathfrak{g}^{\prime}$, and $d \omega=0$ on $\mathfrak{g}^{\prime}$. From Lemma 4.2 we get that $\mathfrak{g}^{\prime}$ is abelian.

Since $\operatorname{ad}_{A}$ is symmetric and $d \omega(A, X, J Y)=\theta \wedge \omega(A, X, J Y)$, we have that

$$
2\langle[A, X], Y\rangle=-\langle X, Y\rangle, \quad \text { for } X, Y \in \mathfrak{g}^{\prime} .
$$

Therefore $[A, X]=-\frac{1}{2} X$ for all $X \in \mathfrak{g}^{\prime}$. Setting $B=J A$, we obtain $[A, B]=0$, thus $\mathfrak{g}=\mathbb{R}^{2} \ltimes \mathbb{R}^{2 n}$ where $\left.\operatorname{ad}_{A}\right|_{\mathbb{R}^{2 n}}=-\frac{1}{2}$ Id and $\left.\operatorname{ad}_{B}\right|_{\mathbb{R}^{2 n}}=J \operatorname{ad}_{A}=-\frac{1}{2} J$.

Corollary 4.4. There exists no unimodular Lie algebra $\mathfrak{g}$ with an l.c.K. structure $(J,\langle\cdot, \cdot\rangle)$ such that $J$ is a bi-invariant complex structure.

Let $M$ be a compact complex parallelizable manifold. According to [28], $M$ may be written as a quotient $\Gamma \backslash G$, where $G$ is a simply connected complex Lie group and $\Gamma$ is a discrete subgroup. Note that according to [15], $G$ must be unimodular. Let $\pi: G \rightarrow M$ denote the holomorphic projection. 
Corollary 4.5. With notation as above, $M$ does not admit any l.c.K. metric $g$ compatible with its holomorphic structure such that $\pi^{*} g$ is a left-invariant metric on $G$.

Remark 4.6. In [10], it is proved more generally that a compact complex parallelizable manifold does not admit any l.c.K. metric compatible with its holomorphic structure.

\section{L.C.K. STRUCTURES With ABELIAN COMPLEX STRUCTURE}

In this section we consider a Lie algebra equipped with an l.c.K. structure such that its complex structure is abelian. Our aim is to prove the following result, where we show that the only unimodular Lie algebras that admit such metrics are the product of a Heisenberg Lie algebra by $\mathbb{R}$, and the l.c.K. structure is in fact Vaisman. From now on we assume that the Lie algebras we work with are at least 4-dimensional.

Before stating the main result, we consider the following variation of Example 3.2. Recall that $\mathbb{R} \times \mathfrak{h}_{2 n+1}$ has a basis $\left\{X_{1}, \ldots, X_{n}, Y_{1}, \ldots, Y_{n}, Z_{1}, Z_{2}\right\}$ such that $\left[X_{i}, Y_{i}\right]=Z_{1}$ for $i=1, \ldots, n$, and that this Lie algebra admits an abelian complex structure $J_{0}$ given by $J_{0} X_{i}=Y_{i}, J_{0} Z_{1}=-Z_{2}$. For any $\lambda>0$, consider the metric $\langle\cdot, \cdot\rangle_{\lambda}$ such that the basis above is orthogonal, with $\left|X_{i}\right|=\left|Y_{i}\right|=1$ but $\left|Z_{1}\right|^{2}=\left|Z_{2}\right|^{2}=\frac{1}{\lambda}$. It is easy to see (just as in Example 3.2) that $\left(J_{0},\langle\cdot, \cdot\rangle_{\lambda}\right.$ ) is an l.c.K. structure, in fact, it is Vaisman. Furthermore, the metrics $\langle\cdot, \cdot\rangle_{\lambda}$ are pairwise non-isometric, since the scalar curvature of $\langle\cdot, \cdot\rangle_{\lambda}$ is $-\frac{n \lambda^{2}}{2}$.

Theorem 5.1. Let $(J,\langle\cdot, \cdot\rangle)$ be an l.c.K. structure on $\mathfrak{g}$ with abelian complex structure J. If $\mathfrak{g}$ is unimodular then $\mathfrak{g} \simeq \mathbb{R} \times \mathfrak{h}_{2 n+1}$, where $\mathfrak{h}_{2 n+1}$ is the $(2 n+1)$-dimensional Heisenberg Lie algebra, and $(J,\langle\cdot, \cdot\rangle)$ is equivalent to $\left(J_{0},\langle\cdot, \cdot\rangle_{\lambda}\right)$ for some $\lambda>0$.

We will provide the proof of this theorem in a series of results. Recall from (8) that

$$
\mathfrak{g}=\operatorname{span}\{A\} \oplus \operatorname{ker} \theta,
$$

where $A \in(\operatorname{ker} \theta)^{\perp}$ such that $\theta(A)=1$.

Lemma 5.2. The endomorphism $\operatorname{ad}_{A}: \mathfrak{g} \rightarrow \mathfrak{g}$ is symmetric.

Proof. It is an immediate consequence of Lemma 3.4.

In particular $\left.\operatorname{ad}_{A}\right|_{\operatorname{ker} \theta}: \operatorname{ker} \theta \rightarrow \operatorname{ker} \theta$ is symmetric, therefore it is diagonalizable and thus we get the following decomposition:

$$
\operatorname{ker} \theta=\sum_{\lambda \in S} \mathfrak{g}_{\lambda}
$$

where $S$ is the spectrum of $\left.\operatorname{ad}_{A}\right|_{\operatorname{ker} \theta}$ and $\mathfrak{g}_{\lambda}$ is the eigenspace associated with the eigenvalue $\lambda$.

According to Lemma 2.5 (iii) the codimension of $[\mathfrak{g}, \mathfrak{g}]$ is at least 2. Therefore $\mathfrak{g}_{0} \neq\{0\}$, that is $0 \in S$. Hence we obtain the following orthogonal decomposition:

$$
\mathfrak{g}=\mathbb{R} A \oplus \mathfrak{g}_{0} \oplus \sum_{\lambda \in S^{*}} \mathfrak{g}_{\lambda},
$$

where $S^{*}:=S-\{0\}$. Note that the Jacobi's identity together with the fact that $\mathfrak{g}^{\prime}$ is abelian imply that:

- $\mathfrak{g}_{\lambda}$ is an ideal for $\lambda \in S^{*}$.

- $\mathfrak{g}_{0}$ is a subalgebra. 
Now we consider $\mathfrak{g}_{0}^{\prime}=\left[\mathfrak{g}_{0}, \mathfrak{g}_{0}\right]$ and $\left(\mathfrak{g}_{0}^{\prime}\right)^{\perp}$, its orthogonal complement in $\mathfrak{g}_{0}$, that is,

$$
\mathfrak{g}_{0}=\mathfrak{g}_{0}^{\prime} \oplus\left(\mathfrak{g}_{0}^{\prime}\right)^{\perp} .
$$

Note also that $\mathfrak{g}^{\prime}=\mathfrak{g}_{0}^{\prime} \oplus \sum_{\lambda \in S^{*}} \mathfrak{g}_{\lambda}$.

For any $X, Y \in \operatorname{ker} \theta$, we compute

$$
\begin{aligned}
d \omega(A, X, Y) & =-\omega([A, X], Y)-\omega([X, Y], A)-\omega([Y, A], X) \\
& =-\langle J[A, X], Y\rangle-\langle J[X, Y], A\rangle-\langle J[Y, A], X\rangle \\
& =\langle[A, X], J Y\rangle+\langle[X, Y], J A\rangle+\langle[Y, A], J X\rangle \\
\theta \wedge \omega(A, X, Y) & =\theta(A) \omega(X, Y)+\theta(X) \omega(Y, A)+\theta(Y) \omega(A, X) \\
& =\langle J X, Y\rangle .
\end{aligned}
$$

Therefore we have

$$
\langle[A, X], J Y\rangle+\langle[X, Y], J A\rangle+\langle[Y, A], J X\rangle=\langle J X, Y\rangle,
$$

for all $X, Y \in \operatorname{ker} \theta$. From this we get three equations that will be important later:

$$
\begin{gathered}
\langle[X, Y], J A\rangle=\langle J X, Y\rangle, \text { for } X, Y \in \mathfrak{g}_{0} . \\
(\lambda+\mu+1)\langle J X, Y\rangle=0, \text { for } X \in \mathfrak{g}_{\lambda}, Y \in \mathfrak{g}_{\mu} \text { and } \lambda, \mu \in S^{*} . \\
\langle[X, Y], J A\rangle=(\mu+1)\langle J X, Y\rangle, \text { for } X \in \mathfrak{g}_{0}, Y \in \mathfrak{g}_{\mu}, \mu \in S^{*} .
\end{gathered}
$$

Lemma 5.3. $J\left(\mathfrak{g}_{0}^{\prime}\right) \subset \mathbb{R} A \oplus\left(\mathfrak{g}_{0}^{\prime}\right)^{\perp}$.

Proof. If $X, Y \in \mathfrak{g}_{0}$ we can write $J[X, Y]=a A+Z_{0}+\sum_{\lambda \in S^{*}} Z_{\lambda}$ with $a \in \mathbb{R}, Z_{0} \in \mathfrak{g}_{0}$ and $Z_{\lambda} \in \mathfrak{g}_{\lambda}$. Then $[A, J[X, Y]]=\left[A, \sum_{\lambda \in S^{*}} Z_{\lambda}\right]=\sum_{\lambda \in S^{*}} \lambda Z_{\lambda}$.

On the other hand, from (12), $J A=X_{0}+\sum_{\lambda \in S^{*}} X_{\lambda}$, with $X_{0} \in \mathfrak{g}_{0}$ y $X_{\lambda} \in \mathfrak{g}_{\lambda}$ for all $\lambda \in S^{*}$. Then

$$
[A, J[X, Y]]=-[J A,[X, Y]]=-\left[X_{0}+\sum_{\lambda \in S^{*}} X_{\lambda},[X, Y]\right]=-\left[X_{0},[X, Y]\right] \in \mathfrak{g}_{0}
$$

since $\mathfrak{g}_{0}$ is subalgebra and $\mathfrak{g}^{\prime}$ is abelian. Therefore $Z_{\lambda}=0$ for all $\lambda \in S^{*}$.

Moreover, it follows from (13) and the fact that $\mathfrak{g}^{\prime}$ is abelian that $J\left(\mathfrak{g}_{0}^{\prime}\right)$ and $\mathfrak{g}_{0}^{\prime}$ are orthogonal. Then we must have $Z_{0} \in\left(\mathfrak{g}_{0}^{\prime}\right)^{\perp}$, and this implies the result.

Now, we define $\Lambda \subset S^{*}$ in the following way: $\lambda \in \Lambda$ if and only if there is not any $\lambda^{\prime} \in S^{*}$ such that $\lambda+\lambda^{\prime}+1=0$, or equivalently, $\Lambda=\left\{\lambda \in S^{*}:-(\lambda+1) \notin S^{*}\right\}$. Note that $\lambda \notin \Lambda$ if and only if $-(\lambda+1) \notin \Lambda$.

Lemma 5.4. Let $\lambda \in S^{*}$. Then,

(i) if $\lambda \in \Lambda$ then $J\left(\mathfrak{g}_{\lambda}\right) \subset \mathbb{R} A \oplus\left(\mathfrak{g}_{0}^{\prime}\right)^{\perp}$.

(ii) if $\lambda \in \Lambda^{c}$ then $J\left(\mathfrak{g}_{\lambda}\right) \subset \mathbb{R} A \oplus\left(\mathfrak{g}_{0}^{\prime}\right)^{\perp} \oplus \mathfrak{g}_{\lambda^{\prime}}$, where $\lambda+\lambda^{\prime}+1=0$.

Proof. (i) If $\lambda \in \Lambda$, from (14) we have that $J\left(\mathfrak{g}_{\lambda}\right)$ is orthogonal to $\mathfrak{g}_{\mu}$ for all $\mu \in S^{*}$, and therefore $J\left(\mathfrak{g}_{\lambda}\right) \subset \mathbb{R} A \oplus \mathfrak{g}_{0}$. Moreover, for $X_{\lambda} \in \mathfrak{g}_{\lambda}, Y \in \mathfrak{g}_{0}^{\prime}$, it follows from Lemma 5.3 that $\left\langle J X_{\lambda}, Y\right\rangle=$ $-\left\langle X_{\lambda}, J Y\right\rangle=0$. This proves (i), and in a similar way we prove (ii). 
Let $\mathfrak{h}$ be the orthogonal complement of $\mathfrak{g}^{\prime}+J \mathfrak{g}^{\prime}$ in $\mathfrak{g}$. Note that $\mathfrak{h}$ is $J$-invariant. Thus, we can write

$$
\mathfrak{g}=\left(\mathfrak{g}^{\prime}+J \mathfrak{g}^{\prime}\right) \oplus \mathfrak{h}
$$

We will show that this orthogonal complement $\mathfrak{h}$ is non-zero. We begin with an auxiliary result.

Lemma 5.5. $\mathfrak{g}^{\prime} \cap J \mathfrak{g}^{\prime}=\sum_{\Lambda^{c}} \mathfrak{g}_{\lambda} \cap J\left(\sum_{\Lambda^{c}} \mathfrak{g}_{\lambda}\right)$.

Proof. Given $Y \in \mathfrak{g}^{\prime} \cap J \mathfrak{g}^{\prime}$ then $Y=J Z$ for $Z \in \mathfrak{g}^{\prime}$. Since $\mathfrak{g}^{\prime}=\mathfrak{g}_{0}^{\prime} \oplus \sum_{\lambda \in S^{*}} \mathfrak{g}_{\lambda}$, we can write

$$
Y=Y_{0}+\sum_{\lambda \in S^{*}} Y_{\lambda}, \quad Z=Z_{0}+\sum_{\lambda \in S^{*}} Z_{\lambda}
$$

with $Y_{0}, Z_{0} \in \mathfrak{g}_{0}^{\prime}$ and $Y_{\lambda}, Z_{\lambda} \in \mathfrak{g}_{\lambda}$. Then $J Z=J Z_{0}+\sum_{\lambda \in S^{*}} J Z_{\lambda}$. From Lemma 5.3 and Lemma 5.4 we obtain $J Z \in \mathbb{R} A \oplus\left(\mathfrak{g}_{0}^{\prime}\right)^{\perp} \oplus \sum_{\lambda \in \Lambda^{c}} \mathfrak{g}_{\lambda}$. Since $J Z=Y \in \mathfrak{g}_{0}^{\prime} \oplus \sum_{\lambda \in S^{*}} \mathfrak{g}_{\lambda}$, we get $Y_{0}=0$ and $Y_{\lambda}=0$ for all $\lambda \in \Lambda$, therefore $Y \in \sum_{\lambda \in \Lambda^{c}} \mathfrak{g}_{\lambda}$. In the same way, $Z \in \sum_{\lambda \in \Lambda^{c}} \mathfrak{g}_{\lambda}$, and therefore $\mathfrak{g}^{\prime} \cap J \mathfrak{g}^{\prime} \subset \sum_{\Lambda^{c}} \mathfrak{g}_{\lambda} \cap J\left(\sum_{\Lambda^{c}} \mathfrak{g}_{\lambda}\right)$. The other inclusion is clear.

Lemma 5.6. With notation as above, $\mathfrak{h} \neq 0$.

Proof. If we suppose that $\mathfrak{h}=\{0\}$ we get from (16) that

$$
\mathfrak{g}=\mathfrak{g}^{\prime}+J \mathfrak{g}^{\prime} .
$$

Claim: $\mathfrak{g}^{\prime} \cap J \mathfrak{g}^{\prime}=\{0\}$.

Indeed, according to Lemma 5.5 and to Lemma 2.5 (ii) we have $\mathfrak{g}^{\prime} \cap J \mathfrak{g}^{\prime}=\sum_{\Lambda^{c}} \mathfrak{g}_{\lambda} \cap J\left(\sum_{\Lambda^{c}} \mathfrak{g}_{\lambda}\right) \subset \mathfrak{z}(\mathfrak{g})$. Given $Y \in \mathfrak{g}^{\prime} \cap J \mathfrak{g}^{\prime}$, it can be written as $Y=\sum_{\lambda \in \Lambda^{c}} Y_{\lambda}$. Then $0=[A, Y]=\sum_{\lambda \in \Lambda^{c}} \lambda Y_{\lambda}$, and therefore $Y=0$. This proves the claim.

As a consequence, we have the orthogonal decomposition

$$
\mathfrak{g}=\mathfrak{g}^{\prime} \oplus J \mathfrak{g}^{\prime} .
$$

According to [2, Corollary 3.3], the Lie bracket on $\mathfrak{g}$ induces a structure of commutative associative algebra on $\mathfrak{g}^{\prime}$ given by $X * Y=[J X, Y]$. Furthermore if $\mathcal{A}$ denotes the commutative associative algebra $\left(\mathfrak{g}^{\prime}, *\right)$, then $\mathcal{A}^{2}=\mathcal{A}$ and $\mathfrak{g}$ is holomorphically isomorphic to $\mathfrak{a f f}(\mathcal{A})$ with its standard complex structure (see Section 2.2). Since $\mathfrak{g}$ is unimodular, it follows from Lemma 2.6 that $\mathcal{A}$ is nilpotent. This is a contradiction with the fact that $\mathcal{A}^{2}=\mathcal{A}$ and therefore it must be $\mathfrak{h} \neq\{0\}$. 
Since $A$ is orthogonal to $\mathfrak{g}^{\prime}$, we have that $J A$ is orthogonal to $J \mathfrak{g}^{\prime}$. More precisely, we have

Lemma 5.7. $J A \in \mathfrak{g}^{\prime}$.

Proof. Let $\mathfrak{u}$ be the orthogonal complement of $\mathfrak{g}^{\prime} \cap J \mathfrak{g}^{\prime}$ in $\mathfrak{g}^{\prime}$, that is

$$
\mathfrak{g}^{\prime}=\mathfrak{u} \oplus\left(\mathfrak{g}^{\prime} \cap J \mathfrak{g}^{\prime}\right) .
$$

Since $\mathfrak{g}^{\prime} \cap J \mathfrak{g}^{\prime}$ is $J$-invariant we have

$$
J \mathfrak{g}^{\prime}=J \mathfrak{u} \oplus\left(\mathfrak{g}^{\prime} \cap J \mathfrak{g}^{\prime}\right),
$$

and therefore

$$
\mathfrak{g}^{\prime}+J \mathfrak{g}^{\prime}=\mathfrak{u} \oplus\left(\mathfrak{g}^{\prime} \cap J \mathfrak{g}^{\prime}\right) \oplus J \mathfrak{u} .
$$

As $J A$ is orthogonal to $J \mathfrak{g}^{\prime}$, it follows from (16) that $J A=U+\beta$ for some $U \in \mathfrak{u}$ and $\beta \in \mathfrak{h}$. Since $\mathfrak{u} \subset \mathfrak{g}^{\prime}$, the lemma will follow if we prove $\beta=0$.

Now, for any $X \in \mathfrak{g}$ such that $\langle A, X\rangle=\langle J A, X\rangle=0$, we compute

$$
\begin{aligned}
d \omega(J \beta, X, J X) & =\theta \wedge \omega(J \beta, X, J X) \\
-\langle[J \beta, X], X\rangle+\langle[X, J X],-\beta]\rangle+\langle[J X, J \beta], J X\rangle & =\frac{\langle A, J \beta\rangle}{|A|^{2}}|X|^{2} \\
\langle[J \beta, X], X\rangle+\langle[J \beta, J X], J X\rangle & =\frac{|\beta|^{2}}{|A|^{2}}|X|^{2} .
\end{aligned}
$$

Moreover $\langle[J \beta, A], A\rangle=0$ and $\langle[J \beta, J A], J A\rangle=0$, due to Lemma 3.4 and the fact that $\mathfrak{h}$ is orthogonal to $\mathfrak{g}^{\prime}$. Let $\left\{X_{1}, J X_{1}, \ldots, X_{r}, J X_{r}\right\}$ be an orthonormal basis of $W$, where $W$ is given in (9). Note that $r \geq 1$ since $\operatorname{dim} \mathfrak{g} \geq 4$. We compute next $\operatorname{tr}\left(\operatorname{ad}_{J \beta}\right)$, taking into account (17):

$$
\begin{aligned}
\operatorname{tr}\left(\operatorname{ad}_{J \beta}\right) & =\frac{1}{|A|^{2}}\langle[J \beta, A], A\rangle+\frac{1}{|A|^{2}}\langle[J \beta, J A], J A\rangle+\sum_{j=1}^{r}\left\langle\left[J \beta, X_{j}\right], X_{j}\right\rangle+\left\langle\left[J \beta, J X_{j}\right], J X_{j}\right\rangle \\
& =\frac{|\beta|^{2}}{|A|^{2}} \sum_{j=1}^{r}|X|^{2} .
\end{aligned}
$$

Since $\mathfrak{g}$ is unimodular, it follows that $\beta=0$.

Remark 5.8. It follows from Lemma 5.7 and (16) that if $H \in \mathfrak{h}$, then $H$ is orthogonal to $A$ and $J A$.

Lemma 5.9. If $H \in \mathfrak{h}$, then

(i) $\langle[H, J H], J A\rangle=|H|^{2}$,

(ii) $|[H, J H]|^{2}=\frac{|H|^{4}}{|A|^{2}}$.

Proof. For $H \in \mathfrak{h}$, we compute first

$$
\begin{aligned}
d \omega(A, H, J H) & =\theta \wedge \omega(A, H, J H) \\
\left\langle[A, H], J^{2} H\right\rangle+\langle[H, J H], J A\rangle+\langle[J H, A], J H\rangle & =|H|^{2}-\frac{\langle A, H\rangle^{2}}{|A|^{2}}-\frac{\langle J A, H\rangle^{2}}{|A|^{2}} \\
\langle[H, J H], J A\rangle & =|H|^{2},
\end{aligned}
$$

since $\mathfrak{h}$ is $J$-invariant and orthogonal to $\mathfrak{g}^{\prime}$. This proves (i). 
Now we compute

$$
\begin{aligned}
d \omega(J[H, J H], H, J H) & =\theta \wedge \omega(J[H, J H], H, J H) \\
-|[H, J H]|^{2} & =\frac{\langle A, J[H, J H]\rangle}{|A|^{2}}|H|^{2} \\
|[H, J H]|^{2} & =\frac{|H|^{4}}{|A|^{2}},
\end{aligned}
$$

where we used (i) for the last equality. This proves (ii).

Lemma 5.10. If $H \in \mathfrak{h}$, then

(i) $[H, J H]=\frac{|H|^{2}}{|A|^{2}} J A$,

(ii) $\left[H, \mathfrak{g}_{0}^{\prime}\right]=0$,

(iii) $\left[H, \mathfrak{g}_{\lambda}\right]=0$ for all $\lambda \in S^{*}-\left\{-\frac{1}{2}\right\}$,

(iv) $[H, Y]=0$ for all $Y \in \mathfrak{h}$ such that $\langle Y, J H\rangle=0$.

Proof. (i) Using Lemma 5.9 and the Cauchy-Schwarz inequality we obtain

$$
|H|^{4}=\langle[H, J H], J A\rangle^{2} \leq|[H, J H]|^{2}|A|^{2}=\frac{|H|^{4}}{|A|^{2}}|A|^{2}=|H|^{4},
$$

so that we have equality everywhere and therefore for all $H \in \mathfrak{h}$ there exists $c(H) \in \mathbb{R}$ such that

$$
[H, J H]=c(H) J A \text {. }
$$

From Lemma 5.9 (ii) again we get that $|H|^{2}=c(H)|A|^{2}$, and therefore $[H, J H]=\frac{|H|^{2}}{|A|^{2}} J A$ for all $H \in \mathfrak{h}$.

Both (ii) and (iii) will follow from the next computation. Given $H \in \mathfrak{h}, X, Y \in \mathfrak{g}^{\prime}$ we compute

$$
\begin{aligned}
d \omega(H, X, J Y) & =-\omega([H, X], J Y)-\omega([X, J Y], H)-\omega([J Y, H], X) \\
& =-\langle J[H, X], J Y\rangle-\langle J[X, J Y], H\rangle-\langle J[J Y, H], X\rangle \\
& =-\langle[H, X], Y\rangle+\langle[X, J Y], J H\rangle+\langle[J Y, H], J X\rangle \\
& =-\langle[H, X], Y\rangle+\langle[J Y, H], J X\rangle,
\end{aligned}
$$

since $\mathfrak{h}$ is $J$-invariant and orthogonal to $\mathfrak{g}^{\prime}$. On the other hand we have

$$
\begin{aligned}
\theta \wedge \omega(H, X, J Y) & =\theta(H) \omega(X, J Y)+\theta(X) \omega(J Y, H)+\theta(J Y) \omega(H, X) \\
& =\frac{\langle A, H\rangle}{|A|^{2}}\langle X, Y\rangle-\frac{\langle A, X\rangle}{|A|^{2}}\langle Y, H\rangle+\frac{\langle A, J Y\rangle}{|A|^{2}}\langle J H, X\rangle \\
& =0,
\end{aligned}
$$

since $\langle H, A\rangle=0$ and $\left\langle\mathfrak{h}, \mathfrak{g}^{\prime}\right\rangle=0$. Therefore we get $\langle[H, X], Y\rangle=\langle[J Y, H], J X\rangle$. In particular, if we take $Y=[H, X]$ we obtain

$$
|[H, X]|^{2}=\langle[J[H, X], H], J X\rangle .
$$

(ii) If $X \in \mathfrak{g}_{0}^{\prime}$ it follows from Lemma 5.3 that $J X \in \mathbb{R} A \oplus\left(\mathfrak{g}_{0}^{\prime}\right)^{\perp}$. Since $[J[H, X], H] \in \mathfrak{g}^{\prime}$ we get from (18) that $|[H, X]|^{2}=0$.

(iii) If $X_{\lambda} \in \mathfrak{g}_{\lambda}, \lambda \in \Lambda$, it follows from Lemma 5.4 (i) that $J X \in \mathbb{R} A \oplus\left(\mathfrak{g}_{0}^{\prime}\right)^{\perp}$. In the same way as above we get $|[H, X]|^{2}=0$.

However, if $X_{\lambda} \in \mathfrak{g}_{\lambda}, \lambda \in \Lambda^{c}$ and $\lambda \neq-\frac{1}{2}$ from Lemma 5.4 (ii) we obtain that $J X \in \mathbb{R} A \oplus$ $\left(\mathfrak{g}_{0}^{\prime}\right)^{\perp} \oplus \mathfrak{g}_{\lambda^{\prime}}$, where $\lambda^{\prime}=-\lambda-1$ and $\lambda^{\prime} \neq \lambda$ since $\lambda \neq-\frac{1}{2}$. On the other hand $[J[H, X], H]=$ $-[[H, X], J H] \in \mathfrak{g}_{\lambda}$ since $\mathfrak{g}_{\lambda}$ is an ideal. Therefore from (14) and (18) we get $|[H, X]|^{2}=0$. 
(iv) Finally, we calculate $[H, Y]$ for $Y \in \mathfrak{h}$ such that $\langle Y, J H\rangle=0$.

$$
\begin{aligned}
d \omega(J[H, Y], H, Y) & =\langle[J[H, Y], H], J Y\rangle-|[H, Y]|^{2}+\langle[Y, J[H, Y]], J H\rangle \\
& =-|[H, Y]|^{2},
\end{aligned}
$$

since $\mathfrak{h}$ is $J$-invariant and orthogonal to $\mathfrak{g}^{\prime}$. On the other hand

$$
\theta \wedge \omega(J[H, Y], H, Y)=\frac{\langle A, J[H, Y]\rangle}{|A|^{2}}\langle J H, Y\rangle+\frac{\langle A, H\rangle}{|A|^{2}}\langle J Y, J[H, Y]\rangle-\frac{\langle A, Y\rangle}{|A|^{2}}\langle[H, Y], H\rangle=0,
$$

since $A$ is orthogonal to $\mathfrak{h}$ and $\langle J H, Y\rangle=0$. Therefore $[H, Y]=0$.

Proposition 5.11. With notation as above, we have:

(i) $A, J A \in \mathfrak{z}(\mathfrak{g})$,

(ii) $\mathfrak{g}=\mathfrak{g}^{\prime} \oplus J \mathfrak{g}^{\prime} \oplus \mathfrak{h}$, an orthogonal sum.

Proof. (i) Let $\lambda \in S^{*}-\left\{-\frac{1}{2}\right\}$ and take $H \in \mathfrak{h}, H \neq 0$ and $X_{\lambda} \in \mathfrak{g}_{\lambda}$. Lemma 5.10 (i) implies that

$$
\left[[H, J H], J X_{\lambda}\right]=\frac{|H|^{2}}{|A|^{2}}\left[J A, J X_{\lambda}\right]=\frac{|H|^{2}}{|A|^{2}} \lambda X_{\lambda},
$$

whereas Lemma 5.10 (ii) and the fact that $\mathfrak{h}$ is $J$-invariant imply that

$$
\left[[H, J H], J X_{\lambda}\right]=-\left[\left[J H, J X_{\lambda}\right], H\right]-\left[\left[J X_{\lambda}, H\right], J H\right]=0 .
$$

Then $X_{\lambda}=0$ and therefore $S^{*}-\left\{-\frac{1}{2}\right\}=\emptyset$. If $-\frac{1}{2} \in S^{*}$ then it is the only eigenvalue in $S^{*}$, hence $\mathfrak{g}$ is not unimodular, that is a contradiction. As a consequence, $S^{*}=\emptyset$, that is, $S=\{0\}$, or equivalently, $A \in \mathfrak{z}(\mathfrak{g})$. It follows from Lemma 2.5 that $J A \in \mathfrak{z}(\mathfrak{g})$ too.

(ii) It follows from Lemma 5.5 that $\mathfrak{g}^{\prime} \cap J \mathfrak{g}^{\prime}=\{0\}$. Therefore

$$
\mathfrak{g}=\mathfrak{g}^{\prime} \oplus J \mathfrak{g}^{\prime} \oplus \mathfrak{h}
$$

where $\mathfrak{g}^{\prime}=\mathfrak{g}_{0}^{\prime}$. Moreover, this decomposition is orthogonal, because of Lemma 5.3.

Remark 5.12. If $(\mathfrak{g}, J,\langle\cdot, \cdot\rangle)$ is Vaisman with $J$ abelian, it is much easier to show that $A, J A \in \mathfrak{z}(\mathfrak{g})$. Indeed, from Lemma 5.2 and Lemma 3.5 we have that $\operatorname{ad}_{A}$ is symmetric and skew-symmetric, so $A \in \mathfrak{z}(\mathfrak{g})$. Then $J$ abelian implies that $J A \in \mathfrak{z}(\mathfrak{g})$ too.

Proof of Theorem 5.1. Recall that we have the following orthogonal decompositions of $\mathfrak{g}$,

$$
\mathfrak{g}=\operatorname{span}\{A\} \oplus \mathfrak{g}_{0}=\mathfrak{g}^{\prime} \oplus J \mathfrak{g}^{\prime} \oplus \mathfrak{h},
$$

with $J A \in \mathfrak{g}^{\prime}$, where $\mathfrak{g}^{\prime}$ and $J \mathfrak{g}^{\prime}$ are abelian subalgebras. Since $\mathfrak{g}_{0}^{\prime}=\mathfrak{g}^{\prime}$, it follows from Lemma 5.10 that $\left[\mathfrak{h}, \mathfrak{g}^{\prime}\right]=\left[\mathfrak{h}, J \mathfrak{g}^{\prime}\right]=0$ and, moreover, the Lie bracket on $\mathfrak{h}$ is also determined. In order to characterize completely the Lie bracket on $\mathfrak{g}$, we only need to consider $\left[\mathfrak{g}^{\prime}, J \mathfrak{g}^{\prime}\right]$, that is, the brackets $[X, J Y]$ for $X, Y \in \mathfrak{g}^{\prime}$. Since $A, J A \in \mathfrak{z}(\mathfrak{g})$ we may assume that $X, Y$ are orthogonal to $J A$.

Proposition 5.13. Using the previous notation, we have that:

(i) $[X, J Y]=0$ for $\langle X, Y\rangle=0$,

(ii) $[X, J X]=\frac{|X|^{2}}{|A|^{2}} J A$. 
Proof. We calculate

$$
d \omega(J[X, J Y], X, J Y)=-\langle[J[X, J Y], X], Y\rangle-\langle[X, J Y],[X, J Y]\rangle+\langle[J Y, J[X, J Y]], J X\rangle .
$$

Since $\mathfrak{g}^{\prime}$ and $J \mathfrak{g}^{\prime}$ are orthogonal, we get $\langle[J Y, J[X, J Y]], J X\rangle=0$. From Jacobi identity and the fact that $\mathfrak{g}^{\prime}$ is abelian we have that

$$
[J[X, J Y], X]=-[[X, J Y], J X]=[[J Y, J X], X]+[[J X, X], J Y]=[[J X, X], J Y] .
$$

Therefore $d \omega(J[X, J Y], X, J Y)=-|[X, J Y]|^{2}+\left\langle\operatorname{ad}_{J[J X, X]} Y, Y\right\rangle$. On the other hand,

$$
\theta \wedge \omega(J[X, J Y], X, J Y)=\frac{\langle A, J[X, J Y]\rangle}{|A|^{2}}\langle X, Y\rangle-\frac{\langle A, X\rangle}{|A|^{2}}\langle Y, J[X, J Y]\rangle-\frac{\langle A, J Y\rangle}{|A|^{2}}\langle[X, J Y], X\rangle .
$$

Since $\mathfrak{g}^{\prime}$ and $J \mathfrak{g}^{\prime}$ are orthogonal and the fact that $\langle Y, J A\rangle=0$ we get $\theta \wedge \omega(J[X, J Y], X, J Y)=$ $\frac{\langle A, J[X, J Y]\rangle}{|A|^{2}}\langle X, Y\rangle$. It follows from (13) that $\theta \wedge \omega(J[X, J Y], X, J Y)=-\frac{\langle X, Y\rangle^{2}}{|A|^{2}}$. Therefore

$$
|[X, J Y]|^{2}-\left\langle\operatorname{ad}_{J[J X, X]} Y, Y\right\rangle=\frac{\langle X, Y\rangle^{2}}{|A|^{2}} .
$$

If $\langle Y, X\rangle=0$, we have

$$
\left\langle\operatorname{ad}_{J[J X, X]} Y, Y\right\rangle=|[X, J Y]|^{2} .
$$

If $Y=X$, we get

$$
\left\langle\operatorname{ad}_{J[J X, X]} X, X\right\rangle=|[X, J X]|^{2}-\frac{|X|^{4}}{|A|^{2}} .
$$

Our aim is to calculate $\operatorname{tr}\left(\operatorname{ad}_{J[J X, X]}\right)$ for a fixed $X \in \mathfrak{g}^{\prime}$ and $\langle X, J A\rangle=0$.

It follows from (13) that $\langle[X, J X], J A\rangle=\langle J X, J X\rangle=|X|^{2}$. Then using the Cauchy-Schwarz inequality we obtain

$$
|X|^{4}=|\langle[X, J X], J A\rangle|^{2} \leq|[X, J X]|^{2}|A|^{2}=\left\langle\operatorname{ad}_{J[J X, X]} X, X\right\rangle|A|^{2}+|X|^{4} .
$$

From (19) and (21) we have that $\left\langle\operatorname{ad}_{J[J X, X]} X, X\right\rangle \geq 0$ and $\left\langle\operatorname{ad}_{J[J X, X]} Y, Y\right\rangle \geq 0$ for all $Y \in \mathfrak{g}^{\prime}$, $\langle Y, X\rangle=0$ and $\langle Y, J A\rangle=0$.

We know also that $\operatorname{ad}_{J[J X, X]} J A=0, \operatorname{ad}_{J[J X, X]} J Z=0$ for all $Z \in \mathfrak{g}^{\prime}$ and $\operatorname{ad}_{J[J X, X]} H=0$ for all $H \in \mathfrak{h}$. Therefore

$$
\operatorname{tr}\left(\operatorname{ad}_{J[J X, X]}\right)=\left\langle\operatorname{ad}_{J[J X, X]} X, X\right\rangle+\sum_{\langle Y, X\rangle=0}\left\langle\operatorname{ad}_{J[J X, X]} Y, Y\right\rangle .
$$

Since $\mathfrak{g}$ is unimodular, it must be $\left\langle\operatorname{ad}_{J[J X, X]} Y, Y\right\rangle=0$, and from (19) we get $[X, J Y]=0$ for all $Y \in \mathfrak{g}^{\prime},\langle Y, X\rangle=0$. This proves (i).

(ii) From $(22)$ we have that $\left\langle\operatorname{ad}_{J[J X, X]} X, X\right\rangle=0$. Then it follows from $(21)$ that $[X, J X]=$ $c(X) J A$ with $c(X) \in \mathbb{R}$. From (13) we get $\langle[X, J X], J A\rangle=|X|^{2}$, and this implies that $c(X)=\frac{|X|^{2}}{|A|^{2}}$. Therefore $[X, J X]=\frac{|X|^{2}}{|A|^{2}} J A$ for all $X \in \mathfrak{g}^{\prime}$.

As a consequence, the only non-vanishing brackets on $\mathfrak{g}$ are $[X, J X]=\frac{|X|^{2}}{|A|^{2}} J A$ for $X \in \mathfrak{g}$ with $\langle X, A\rangle=0$ and $\langle X, J A\rangle=0$, so that $\mathfrak{g}^{\prime}=\operatorname{span}\{J A\}$ and $J \mathfrak{g}^{\prime}=\operatorname{span}\{A\}$. Considering an orthonormal basis $\left\{X_{1}, \ldots, X_{n}, Y_{1}, \ldots, Y_{n}\right\}$ of $\mathfrak{h}$, with $J X_{i}=Y_{i}$, we have that the only nonvanishing brackets on $\mathfrak{g}$ are $\left[X_{i}, Y_{i}\right]=\frac{J A}{|A|^{2}}$. Setting $Z_{1}=\frac{J A}{|A|^{2}}, Z_{2}=\frac{A}{|A|^{2}}$, it is clear that $\mathfrak{g}$ is isomorphic to $\mathbb{R} \times \mathfrak{h}_{2 n+1}$ and $(J,\langle\cdot, \cdot\rangle)$ is equivalent to $\left(J_{0},\langle\cdot, \cdot\rangle_{\lambda}\right)$ for $\lambda=|A|$. 
Remark 5.14. On $\mathbb{R} \times \mathfrak{h}_{2 n+1}$ there are $\left[\frac{n}{2}\right]+1$ equivalence classes of complex structures, all of them abelian (see [1, Proposition 2.2]). It follows from the proof of Theorem 5.1 that if $(J,\langle\cdot, \cdot\rangle)$ is an l.c.K. structure on this Lie algebra, then $J$ is equivalent to the complex structure $J_{0}$, so that representatives of only one equivalence class of complex structures may admit l.c.K. metrics (compare [24]).

In terms of solvmanifolds, we can rewrite Theorem 5.1 as follows.

Corollary 5.15. Let $\Gamma \backslash G$ be a compact solvmanifold with an l.c.K. structure induced from a left invariant l.c.K. structure on $G$ with an abelian complex structure, and $G$ simply connected. Then $G$ is isomorphic to $\mathbb{R} \times H_{2 n+1}$, and $\mathbb{R} \times H_{2 n+1}$ has a left-invariant l.c.K. structure induced by $\left(J_{0},\langle\cdot, \cdot\rangle_{\lambda}\right)$ for some $\lambda>0$. In particular, $\Gamma \backslash G$ is a nilmanifold and the l.c.K. structure is Vaisman.

\section{REFERENCES}

[1] A. Andrada, M. L. Barberis, I. G. Dotti, Abelian Hermitian geometry, Diff. Geom. Appl. 30 (2012) 509-519.

[2] A. Andrada, M. L. Barberis, I. G. Dotti, Classification of abelian complex structures on 6-dimensional Lie algebras, J. London Math. Soc. 83 (2011), 232-255.

[3] L. C. de Andrés, L. A. Cordero, M. Fernández, J. J. Mencía, Examples of four dimensional locally conformal Kähler solvmanifolds, Geom. Dedicata 29 (1989), 227-232.

[4] M. L. Barberis, I. G. Dotti, Abelian complex structures on solvable Lie algebras, J. Lie Theory 14 (2004), $25-34$.

[5] M. L. Barberis, I. Dotti, M. Verbitsky, Canonical bundles of complex nilmanifolds, with applications to hypercomplex geometry, Math. Research Letters 16(2) (2009), 331-347.

[6] L. A. Cordero, M. Fernández, M. de LÉon, Compact locally conformal Kähler nilmanifolds, Geom. Dedicata 21 (1986), 187-192.

[7] I. Dotтi, A. Fino, Hyper-Kähler torsion structures invariant by nilpotent Lie groups, Classical Quantum Gravity 19 (2002), 551-562.

[8] S. Dragomir, L. Ornea, Locally conformal Kähler geometry, Progress in Mathematics v. 155, Birkhäuser.

[9] A. Gray, L. Hervella, The sixteen classes of almost Hermitian manifolds and their linear invariants, Ann. Mat. Pura Appl. (4) 123 (1980), 35-58.

[10] K. Hasegawa, Y. Kamishima, Locally conformally Kähler structures on homogeneous spaces, preprint 2011, arXiv:1101.3693.

[11] Y. Kamishima, Note on locally conformal Kähler surfaces, Geom. Dedicata 84 (2001), 115-124.

[12] T. KashiwadA, S. SATo, On harmonic forms in compact locally conformal Kähler manifolds with the parallel Lee form, Ann. Fac. Sci. Univ. Nat. Zä̈re (Kinshasa) Sect. Math.-Phys. 6 (1980), 17-29.

[13] H. KasuYA, Vaisman metrics on solvmanifolds and Oeljeklaus-Toma manifolds, Bull. London Math. Soc. 45 (2013) 15-26.

[14] C. Maclaughlin, H. Pedersen, Y.S.Poon, S. Salamon, Deformation of 2-step nilmanifolds with abelian complex structures, J. London Math. Soc. (2) 73 (2006), 173-193.

[15] J. Milnor, Curvatures of left invariant metrics on Lie groups. Adv. Math. 21 (1976), 293-329.

[16] H. C. LEE, A kind of even dimensional differential geometry and its application to exterior calculus, American J. Math. 65 (1943), 433-438.

[17] P. Libermann, Sur le problème d'equivalence de certaines structures infinitesimales régulières, Annali Mat. Pura Appl. 36 (1954), 27-120.

[18] K. Oeljeklaus, M. Toma, Non-Kähler compact complex manifolds associated to number fields, Ann. Inst. Fourier (Grenoble) 55 (2005), 161-171.

[19] L. Ornea, M. Verbitsky, Topology of locally conformally Kähler manifolds with potential, Int. Math. Res. Not. 2010 (2010), 717-726.

[20] A. P. Petravchuk, Lie algebras decomposable into a sum of an abelian and a nilpotent subalgebra, Ukr. Math. J. 40 (3) (1988), 331-334.

[21] H. SAWAI, Locally conformal Kähler structures on compact nilmanifold with left-invariant complex structures, Geom. Dedicata 125 (2007), 93-101.

[22] H. SAwAI, Locally conformal Kähler structures on compact solvmanifolds, Osaka J. Math. 49 (2012), 1087-1102.

[23] F. Tricerri, Some examples of locally conformal Kähler manifolds, Rend. Sem. Mat. Univ. Politec. Torino 40 (1982), 81-92.

[24] L. UGarte, Hermitian structures on six-dimensional nilmanifolds, Transform. Groups 12 (1), (2007), 175-202. 
[25] I. VAISMAn, Locally conformal Kähler manifolds with parallel Lee form, Rend. Mat., VI Ser. 12 (1979), $263-284$.

[26] I. Vaisman, Generalized Hopf manifolds, Geom. Dedicata 13 (1982), 231-255.

[27] I. Vaisman, On locally conformal almost Kähler manifolds, Israel J. Math. 24 (1976), 338-351.

[28] H. C. Wang, Complex parallelisable manifolds, Proc. Amer. Math. Soc. 5 (1954), 771-776.

E-mail address: andrada@famaf.unc.edu.ar

E-mail address: origlia@famaf.unc.edu.ar

FaMAF-Ciem, Universidad Nacional de Córdoba, Ciudad Universitaria, 5000 Córdoba, Argentina 